\title{
Robotic Pancreaticoduodenectomy: Fad or the Future?
}

\section{Pietro Addeo ${ }^{1 *}$, Daniela Paola Calabrese ${ }^{2}$ and Philippe Bachellier ${ }^{1}$}

${ }^{1}$ HPB Surgery and Liver Transplantation Center, Pole of Digestive Diseases, Liver and Transplantation, Hautepierre Hospital-University Hospital of Strasbourg, Strasbourg University, Strasbourg, France

${ }^{2}$ Department of Surgery, Louis Mourier Hospital, Public Assistance Hopitaux of Paris, University Paris VII, Denis Diderot, Doves, France

\begin{abstract}
The introduction of robotics has opened a new era in general and digestive surgery. Several advanced procedures are now performed with the robotic technique providing superior dexterity to surgeons while maintaining the profile of safety and benefits of minimally invasive surgery for patients. Pancreatic surgery, one of the last area of resistance to minimally invasive surgery, has not been spared by this surgical revolution. An increasing number of studies provided by high volume institutions have proven the safety and feasibility of robotics for pancreatic surgery. Pancreaticoduodenectomy, one of the most advanced surgical procedures in abdominal surgery, represents the most common type of pancreatic resection performed by robotic approach. The safety and feasibility is at present well demonstrated but its advantages over open and laparoscopic surgery and its long term oncologic outcomes are still to be proven in prospective studies.
\end{abstract}

This article reviewed the current experience of robotic pancreaticoduodenectomy reported in the international literature evaluating its safety, feasibility, short and long term outcomes.

The increasing number of reports dealing with this topic proven the growing interest of digestive surgeon for this approach which certainly will constitute the approach of choice for minimally invasive pancreatic surgery in the next future.

Keywords: Robotic surgery; Minimally invasive surgery; Pancreaticoduodenectomy; Pancreatic surgery; Pancreaticoenteric reconstruction; Short term outcomes

\section{Introduction}

Pancreatic surgery represents one of the most advanced fields in the areas of digestive surgery with an historically reported high rate of postoperative morbidity up to $50 \%$ [1].

Surgical approaches to the pancreatic gland require a detailed knowledge of the regional anatomy, dedicated surgical training and adequate skills to dissect tissues and vessels correctly and to perform digestive reconstruction. A large abdominal incision, which can significantly contribute to morbidity and compromise the postoperative immune response after pancreatic resection, is generally required to obtain adequate exposure of the pancreas [2].

In an effort to reduce the high historically rate of postoperative morbidity of pancreatic surgery; twenty years ago laparoscopic surgery was applied to the field of pancreatic surgery. Gagner et al. [3] described the first laparoscopic pancreaticoduodenectomy (PD). However since that time the development of pancreatic surgery has been slow compared with other field of pancreatic surgery. Reasons for that are multifactorial and include the technical challenges of open pancreatic surgery, the intrinsic technical limitations of laparoscopy, the fear of increased morbidity, oncologic concerns, and slow acceptance of laparoscopy among pancreatic surgeons [2].

$\mathrm{PD}$, which represents one the most advanced procedure in the field of abdominal surgery, results very challenging to be performed laparoscopically due to the extensive dissection required around the superior mesenteric vessels and the complexity of digestive reconstruction. Even though some experienced surgeons reported a consistent experience in laparoscopic PD [4-6], the majority of pancreatic surgeons still perform this operation by traditional open surgery. Benefits of minimally invasive surgery have been proven for several digestive procedures and include improved cosmetic, reduced postoperative pain, reduced hospital stay and early recovery [7]. All this benefits of minimally invasive surgery might result of particular importance in case of oncologic diseases leading to an early delivery of adjuvant treatments. Very recently the use of robotic surgical system was introduced in the field of pancreatic surgery, providing a renewed interest for minimally invasive pancreatic surgery [8-13]. The aim of the current article is to review the current experience reported with robotic surgery for PD focusing on feasibility, safety, short and long term outcomes.

\section{Material and Methods}

\section{Data collection}

We searched the Medline (through PubMed) database electronically from 2003 up to and including April 2012. Search terms included the following: pancreas, pancreaticoduodenectomy, duodenopancreatectomy, Whipple's procedure, robotic surgery, resection, and surgery. Terms were searched using both isolated and embedded approaches to identify published articles. Searches were limited to articles in English, those with abstracts and those covering human studies. There were no limits regarding the number of patients included in these studies. For each study, the following parameters were collected: author, period of inclusion, number of patients, Operating

*Corresponding author: Pietro Addeo, HPB Surgery and Liver Transplantation Center, Pole of Digestive Diseases, Liver and Transplantation, Hautepierre Hospital-University Hospital of Strasbourg, Strasbourg University, 1, Avenue Moliere, 67098 Strasbourg, France, E-mail: pietroad@hotmail.com

Received July 16, 2012; Accepted July 18, 2012; Published July 21, 2012

Citation: Addeo P, Calabrese DP, Bachellier P (2012) Robotic Pancreaticoduodenectomy: Fad or the Future? Adv Robot Autom S1:001. doi:10.4172/2168-9695.S1-001

Copyright: ( 2012 Addeo P, et al. This is an open-access article distributed under the terms of the Creative Commons Attribution License, which permits unrestricted use, distribution, and reproduction in any medium, provided the original author and source are credited. 


\begin{tabular}{|c|c|c|c|c|c|c|c|c|c|c|c|}
\hline Author/Year & $\mathbf{N}$ & $\begin{array}{l}\text { OR time } \\
\text { (min) }\end{array}$ & $\begin{array}{l}\text { EBL } \\
(\mathrm{ml})\end{array}$ & $\begin{array}{c}\text { Transfusion } \\
\text { (\%) }\end{array}$ & $\begin{array}{c}\text { Conversion } \\
\text { (\%) }\end{array}$ & $\begin{array}{c}\text { Mortality } \\
(\%)\end{array}$ & $\begin{array}{c}\text { Morbidity } \\
\text { (\%) }\end{array}$ & $\begin{array}{l}\text { Pancreatic } \\
\text { fistula (\%) }\end{array}$ & $\begin{array}{l}\text { LOS } \\
\text { (day) }\end{array}$ & $\operatorname{LN}(n)$ & $\mathrm{R} 1$ rate $(\%)$ \\
\hline Giulianotti et al. [9] 2003 & 8 & 490 & NA & NA & $12.5 \%$ & $12.5 \%$ & $37.5 \%$ & $37.5 \%$ & 20 & NA & NA \\
\hline Kendrick et al. [5] 2010 & 8 & NA & NA & NA & NA & NA & NA & NA & NA & NA & NA \\
\hline Giulianotti et al. [8] 2010 & 60 & 421 & 394 & $10 \%$ & $18.3 \%$ & $3.3 \%$ & NA & $31.7 \%$ & 22 & 18.2 & $8.3 \%$ \\
\hline Narula et al. [14] 2010 & 8 & 420 & NA & NA & $37.5 \%$ & 0 & 0 & 0 & 9.6 & 16 & NA \\
\hline Zhou et al. [15] 2011 & 8 & 718 & 153 & NA & 0 & 0 & $25 \%$ & $25 \%$ & 16.4 & NA & $0 \%$ \\
\hline de Vasconcellos Macedo et al. [16] 2011 & 5 & 640 & NA & 20 & $20 \%$ & 0 & $60 \%$ & $20 \%$ & 25.8 & NA & NA \\
\hline Zureikat et al. [17] 2011 & 24 & 512 & 320 & 20 & NA & 4 & $58.3 \%$ & $21 \%$ & 9 & NA & NA \\
\hline MacKenzie et al. [18] 2011 & NA & 480 & NA & 0 & NA & 0 & NA & NA & 6.2 & 11 & 0 \\
\hline Horiguchi et al. [19] 2011 & 3 & 703 & 118 & 0 & 0 & 0 & $66 \%$ & $33 \%$ & 26 & NA & 0 \\
\hline Chan et al. [20] 2011 & 8 & 478 & 200 & NA & $12.5 \%$ & 0 & $33 \%$ & $25 \%$ & 12 & NA & NA \\
\hline Buchs et al. [21] 2011 & 44 & 444 & 387 & $22.7 \%$ & $4.5 \%$ & $4.5 \%$ & $36.4 \%$ & $18.2 \%$ & 13 & 16.8 & $9.1 \%$ \\
\hline Zeh et al. [22] 2012 & 50 & 568 & 350 & $22 \%$ & $16 \%$ & $2 \%$ & $72 \%$ & $20 \%$ & 10 & $17 \pm 7$ & $11 \%$ \\
\hline Choi et al. [23] 2012 & 1 & 480 & 800 & 0 & 0 & 0 & $100 \%$ & $100 \%$ & 30 & NA & NA \\
\hline Chalikonda et al. [24] 2012 & 30 & 476 & 485 & NA & $10 \%$ & $4 \%$ & 30 & $6.6 \%$ & 9.79 & 13.2 & 0 \\
\hline
\end{tabular}

Table 1: Reported series of robotic pancreatic surgery.

Time (OR), Estimated Blood Loss (EBL), conversion rate, morbidity, rate of pancreatic fistula, mortality, and Length Of Hospital Stay (LOS), number of respected Lymph Nodes (LN) and rate of R1 resection.

\section{Results}

Table 1 shows the results of the current review of robotic PD studies reported. A total of 257 patients were retrieved from the 14 studies available $[5,8,9,14-24]$.

Regarding the feasibility of the approach the overall rate of conversion to open surgery ranged from $0 \%$ to $37.5 \%$. The most common reported causes for conversion were failure to progress, tumoral invasion of the superior mesenteric vessels, pancreatic positive section margin and haemorrhage $[8,22,24]$. Interestingly, Buchs et al. [21] reported a noteworthy rate of $4.5 \%$ conversion to open surgery in 44 consecutive robotic $\mathrm{PD}$. The overall operative time ranged from 420 to 718 minutes with a longer operative time reported mainly at the beginning of the experience. Mortality rate ranged from 0 to $12.5 \%$, with the majority of the author reporting a mortality rate inferior to 5\%. There was one case of intraoperative death reported as a consequence of an intraoperative injury of the portal vein which required an emergent conversion to open surgery [24]. Most of the deaths reported were related to hemorrhagic complication of pancreatic fistula or cardiac event.

The overall morbidity rates ranged from $0 \%$ to $70 \%$, a complication rate similar to that reported in the most recent studies of open PD $[25,26]$. The available studies show a rate of pancreatic fistula after robotic PD ranging between $0 \%$ and $33 \%$, with only the series by Narula et al. [14] reported a noteworthy $0 \%$ rate of pancreatic fistula in eight consecutive patients. A higher rate of fistula formation was observed in some studies that used sclerosis injection for the distal pancreatic stump after PD [8]. However, the rate of clinically significant fistula (Grade B, C) was very limited. Regarding the complications related to pancreatic fistula occurrence, it is noteworthy to underline the rate of bleeding arising from pseudoaneurysms reported in these robotic series was consistent. Buchs et al. [21] reported 3 (6.8\%) of these complications in 44 consecutive cases, Zeh et al. [22] reported 4 (8.0\%) in 50 robotic PDs and Chalikonda et al. [24] reported 1 (3.3\%) in 30 cases. However, the majority of these bleeding events were managed by interventional radiologist with favourable outcomes. The average length of the postoperative hospital stay ranged from 9 to 36 days and depended on the number of patients operated upon and the country in which the study had been performed. Interestingly, Kendrick et al. [5] reported a relatively short median hospital stay of 7 days, and Zeh et al. [22] reported a mean hospital stay of 10 days. Three studies comparing open and robotic PDs have been reported so far. Buchs et al. [21] compared the outcomes of 44 robotic PDs and 39 open PDs in a retrospective unmatched study. These authors showed a significant reduction in the blood loss ( $387 \mathrm{ml}$ vs 827 open, $\mathrm{P}=0.0001)$ and operative time (444 vs $559 \mathrm{~min} ; \mathrm{P}=0.0001)$ in the robotic group. Additionally there was a higher number of lymph nodes harvested in the robotic group (16.8 vs 11; $\mathrm{P}=0.02$ ). Zhou et al. [15] compared 8 robotic $\mathrm{PD}$ to 8 open PD. They found a longer operative time with the robotic approach (718 minutes vs 420 minutes; $\mathrm{P}=0.011$ ). Moreover, they demonstrated a reduced blood loss (153 vs $0.210 \mathrm{ml} ; \mathrm{P}=0.045$ ) and a reduced length of bed stay and hospital stay ( 27.5 vs 96 hours; and 16.4 vs 24.3 days, respectively) in favour of the robotic group. Finally they reported a reduced complication rate associated with a robotic approach $(25 \%$ vs $75 \%$; $\mathrm{P}=0.05)$. Chalikonda et al. [24] in 2012 compared in a casematched study, 30 consecutive robotic PDs to 30 well matched open cases. They reported comparable blood loss, morbidity, mortality, reoperation rate between the two groups, but a longer operative time (476.2 vs $366.4 \mathrm{~min}, \mathrm{P}=0.0005$ ) and a reduced length of hospital stay $(\mathrm{P}=0.043)$ in the robotic group.

Regarding the adequacy of oncologic resection in these studies, few authors have detailed the number of lymph nodes harvested. However, when reported, most of the studies showed the removal of more than 12 lymph nodes, which is considered the gold standard for open pancreaticoduodenectomy [2]. In addition, when comparing the results achieved in the robotic group to the open group, Buchs et al. [21] found an average of 16.8 lymph nodes harvested after a robotic approach and 11 after an open approach $(\mathrm{P}=0.02)$. Similarly only a few studies have reported the rate of positive margins for robotic $\mathrm{PD}$. This rate varied from 0 to $11 \%$, and in all cases the positive margin involved was the retroperitoneal margin, with no involvement at the pancreatic or biliary margins. However, only one study reported the use of a standardized pathological protocol for the assessment of the retroperitoneal margin which is nowadays considered the gold standard in case of pancreatic adenocarcinoma [22].

Finally, considering oncological safety, Giulianotti et al. [8] reported their follow up for malignant lesions. Among 26 patients with adenocarcinoma, 9 were alive with no recurrence at a mean follow up of 16.8 months (range: $8-47$ ). Three were alive with recurrence (local, 
hepatic and pulmonary recurrences). And finally, 5 patients died due to recurrence (after 20,11,11, 9 and 7 months). Among the group with ampullary cancer (15 patients), 10 are alive and disease free at a mean follow up of 50.5 months (range: 8-91). Three patients died, of whom one from recurrence. Of note, no port-site metastases were reported. Kendrick et al. [5] reported 31 patients operated on for pancreatic adenocarcinoma. The mean follow-up for these patients was 7.4 months, with 6 dying as a result of disease recurrence and no port site metastasis were observed.

\section{Discussion}

Pancreatic surgeries have challenged for twenty years the field of minimally invasive surgery. Since the first description by Gagner et al. [3] in 1994 of a laparoscopic PD, several groups attempted to perform this complex operation by laparoscopic approach. Even though some study provided by highly skilled laparoscopic surgeons demonstrated the feasibility of laparoscopy for PD, recently less than 300 cases performed were identified by three different review studies $[2,27,28]$.

Reasons for that are related to the inherent complexity of this operation which entails an extensive dissection around the superior mesenteric vessels followed by a complex reconstruction. These tasks are difficult to be performed laparoscopically due to the limited range of motion of laparoscopic instruments, the two dimensional view and to the steep learning curve.

The introduction of robotic surgery might challenge this concept and might increase the feasibility of a minimally invasive approach for PD.

Robotics seems to improve some steps of this procedure, such as lymphadenectomy and uncinate process dissection, thereby improving the quality of the resection [8]. The microsurgical ability provided by the robotic surgical system also confers superior dexterity when performing biliary and pancreatic reconstruction $[5,8,14]$. Finally the use of robotic systems seems extends the indications for minimally invasive pancreaticoduodenectomy to more complex cases including vascular reconstructions [11].

Over the last three years more than 200 cases were reported by different groups, mainly US centres, with outcomes comparable to open surgery. The largest experience was provided by the University of Illinois at Chicago group [8], who pioneered the use of robotic group for several complex robotic pancreatic surgeries including vascular resection with reconstruction. The feasibility of robotic PD remains well demonstrated with some groups reporting a noteworthy $0 \%$ conversion rate. When present, conversion was most consistently due to technical difficulty or bleeding with one reported death related to this complication. The rate of conversion decreased with the increasing experience of the team and all conversion were described as performed in conditions of safety, challenging the concept of a theoretically difficulties in case of robotic approach.

Regarding the morbidity rate of robotic PD, it appears as comparable to that of the most recent open series. Pancreatic fistula, the most common complication of PD, averaged $20 \%$ in the series reported. Even if this rate appears high, it should be considered that the rate of soft pancreatic parenchyma was high in some studies. It may be because of high number of patients with periampullary disease selected and, due to the fact that one group used at the beginning of their experience Wirsung sclerosis which considerably increases the rate of postoperative pancreatic fistula. Clinical consequences of pancreatic fistulas were similar to open surgery, with some cases of life-threatening bleeding arising from pseudoaneurysm erosion which necessitated endovascular treatment. Delayed gastric emptying and postoperative abscesses were treated in the majority of cases by endoscopic or radiological means. This can attenuate the hypothesis of a decreased morbidity rate for minimally invasive $\mathrm{PD}$, which at the present remains dominated by pancreas related complications. Few studies at the moment compared the open and robotic approach for PD, two were provided from USA and one from China [15,21,24]. Except for one study, two authors reported longer operative time with the robotic approach which consistently decreased over the time. The use of robotic approach was associated with decreased blood loss, transfusion rate, length of hospital stay and overall morbidity. However the rate and clinical gravity of pancreatic complications were similar to open surgery.

As far as oncologic outcomes are concerned, scarce data are currently available to draw firm conclusion. The number of lymph node harvested and the rate of $\mathrm{R} 1$ resection seems appropriate in some of these studies but the length of the follow up is too short and a comparison with the open approach remains anecdotal.

Finally several points remain to be assessed for this new approach. While the number of case performed is growing considerably, only few studies compared robotic PD to open cases which remains the standard approach. Secondly since the current follow up of robotic PD for malignant cases remains short, its use for these cases remains to be validated in large prospective studies. Finally, the cost of this operation are at present considered high due to the use of the robotic system and the need of having two experienced surgeons(consol and patient side surgeons) performing the case.

In conclusion, the robotic approach for PD represents a safe and valid alternative to the open approach in selected cases for well experienced pancreatic surgeon. This approach might provide to patients all the benefits of minimally invasive surgery but not decrease the rate of pancreatic related complications. The long term follow up in case of malignant disease remains to be determined in large prospective studies.

\section{References}

1. Yeo CJ, Cameron JL, Sohn TA, Lillemoe KD, Pitt HA, et al. (1997) Six hundred fifty consecutive pancreaticoduodenectomies in the 1990s: pathology, complications, and outcomes. Ann Surg 226: 248-257.

2. Addeo P, Giulianotti PC (2010) Update on laparoscopic pancreatectomy in 2010. Minerva Chir 65: 655-666.

3. Gagner M, Pomp A (1994) Laparoscopic pylorus-preserving pancreatoduodenectomy. Surg Endosc 8: 408-410.

4. Palanivelu C, Rajan PS, Rangarajan M, Vaithiswaran V, Senthilnathan P, et al (2009) Evolution in techniques of laparoscopic pancreaticoduodenectomy: a decade long experience from a tertiary center. J Hepatobiliary Pancreat Surg 16: 731-740.

5. Kendrick ML, Cusati D (2010) Total laparoscopic pancreaticoduodenectomy feasibility and outcome in an early experience. Arch Surg 145: 19-23.

6. Gumbs AA, Gayet B (2008) The laparoscopic duodenopancreatectomy: the posterior approach. Surg Endosc 22: 539-540.

7. Gagner M, Lacroix A, Bolté E (1992) Laparoscopic adrenalectomy in Cushing's syndrome and pheochromocytoma. N Engl J Med 327: 1033.

8. Giulianotti PC, Sbrana F, Bianco FM, Elli EF, Shah G, et al. (2010) Robotassisted laparoscopic pancreatic surgery: single-surgeon experience. Surg Endosc 24: 1646-1657.

9. Giulianotti PC, Coratti A, Angelini M, Sbrana F, Cecconi S, et al. (2003) Robotics in general surgery: personal experience in a large community hospital. Arch Surg 138: 777-784 
Citation: Addeo P, Calabrese DP, Bachellier P (2012) Robotic Pancreaticoduodenectomy: Fad or the Future? Adv Robot Autom S1:001. doi:10.4172/2168-9695.S1-001

Page 4 of 4

10. Giulianotti PC, Addeo P, Buchs NC, Bianco FM, Ayloo SM (2011) Early experience with robotic total pancreatectomy. Pancreas 40: 311-313

11. Giulianotti PC, Addeo P, Buchs NC, Ayloo SM, Bianco FM (2011) Robotic extended pancreatectomy with vascular resection for locally advanced pancreatic tumors. Pancreas 40: 1264-1270.

12. Giulianotti PC, Sbrana F, Bianco FM, Addeo P, Caravaglios G (2010) Robotassisted laparoscopic middle pancreatectomy. J Laparoendosc Adv Surg Tech A 20: 135-139

13. Addeo P, Marzano E, Nobili C, Bachellier P, Jaeck D, et al. (2011) Robotic central pancreatectomy with stented pancreaticogastrostomy: operative details. Int J Med Robot.

14. Narula VK, Mikami DJ, Melvin WS (2010) Robotic and laparoscopic pancreaticoduodenectomy: a hybrid approach. Pancreas 39: 160-164.

15. Zhou NX, Chen JZ, Liu Q, Zhang X, Wang Z, et al. (2011) Outcomes of pancreatoduodenectomy with robotic surgery versus open surgery. Int $\mathrm{J}$ Med Robot 7: 131-137.

16. de Vasconcellos Macedo AL, Schraibman V, Okazaki S, Mauro F, Epstein $M$, et al. (2011) Treatment of intraductal papillary mucinous neoplasms, neuroendocrine and periampullary pancreatic tumors using robotic surgery: a safe and feasible technique. J Robotic Surg 5: 35-41.

17. Zureikat AH, Nguyen KT, Bartlett DL, Zeh HJ, Moser AJ (2011) Robotic-assisted major pancreatic resection and reconstruction. Arch Surg 146: 256-261.

18. MacKenzie S, Kosari K, Sielaff T, Johnson E (2011) The robotic Whipple: operative strategy and technical considerations. J Robotic Surg 5: 3-9.

19. Horiguchi A, Uyama I, Ito M, Ishihara S, Asano Y, et al. (2011) Robot-assisted laparoscopic pancreatic surgery. J Hepatobiliary Pancreat Sci 18: 488-492.
20. Chan OC, Tang CN, Lai EC, Yang GP, Li MK (2011) Robotic hepatobiliary and pancreatic surgery: a cohort study. J Hepatobiliary Pancreat Sci 18: 471-480.

21. Buchs NC, Addeo P, Bianco FM, Ayloo S, Benedetti E, et al. (2011) Robotic versus open pancreaticoduodenectomy: a comparative study at a single institution. World J Surg 35: 2739-2746.

22. Zeh HJ, Zureikat AH, Secrest A, Dauoudi M, Bartlett D, et al. (2012) Outcomes after robot-assisted pancreaticoduodenectomy for periampullary lesions. Ann Surg Oncol 19: 864-870.

23. Choi SH, Kang CM, Kim DH, Lee WJ, Chi HS (2011) Robotic pylorus preserving pancreaticoduodenectomy with mini-laparotomy reconstruction in patient with ampullary adenoma. J Korean Surg Soc 81: 355-359.

24. Chalikonda S, Aguilar-Saavedra JR, Walsh RM (2012) Laparoscopic roboticassisted pancreaticoduodenectomy: a case-matched comparison with open resection. Surg Endosc.

25. Cameron JL, Riall TS, Coleman J, Belcher KA (2006) One thousand consecutive pancreaticoduodenectomies. Ann Surg 244: 10-15.

26. Winter JM, Cameron JL, Campbell KA, Arnold MA, Chang DC, et al. (2006) 1423 pancreaticoduodenectomies for pancreatic cancer: A single-institution experience. J Gastrointest Surg 10: 1199-1210.

27. Gumbs AA, Rodriguez Rivera AM, Milone L, Hoffman JP (2011) Laparoscopic pancreatoduodenectomy: a review of 285 published cases. Ann Surg Oncol 18: $1335-1341$.

28. Buchs NC, Volonte F, Pugin F, Bucher P, Jung M, et al. (2011) Robotic pancreatic resection: how far can we go? Minerva Chir 66: 603-614 